\title{
Probing the bilayer-monolayer switching of capping agents on Au nanorods and its interaction with guest molecules ${ }^{\S}$
}

\author{
JATISH KUMAR ${ }^{\mathrm{a}, \mathrm{b}}$ and K GEORGE THOMAS ${ }^{\mathrm{a}, *}$ \\ ${ }^{a}$ School of Chemistry, Indian Institute of Science Education and Research Thiruvananthapuram (IISER-TVM), \\ Vithura, Thiruvananthapuram, Kerala 695 551, India \\ ${ }^{\mathrm{b}}$ CSIR -National Institute for Interdisciplinary Science and Technology, Thiruvananthapuram, Kerala 695019 , \\ India \\ E-mail: kgt@iisertvm.ac.in
}

MS received 7 August 2018; revised 25 August 2018; accepted 31 August 2018; published online 26 September 2018

\begin{abstract}
The anisotropic features of Au nanorods make them an attractive nanoscale precursor for the design of higher order nanostructured materials. However, the mode of interaction of various molecular systems on Au nanorods is not well-understood. In the present study, we have employed isothermal titration calorimetry and surface-enhanced Raman scattering for understanding various types of interactions of functional molecules on the surface of gold nanorods. The binding of thiol-bearing analyte molecules is effective with the surface of gold nanorods in acetonitrile-rich solvents and found to be weak in an aqueous medium. The effective interaction of thiol-bearing analyte molecules on nanorods is facilitated by the breakdown of cetyltrimethylammonium bromide bilayer to a monolayer in organic-rich solvent systems, thereby resulting in appreciable signals in isothermal titration calorimetry and surface-enhanced Raman spectra. The electrostatic interaction of analyte molecule is mainly driven by the charge reversal on the surface of Au nanorods on switching the solvent from aqueous to organic medium. Thus, based on isothermal titration calorimetry and surface-enhanced Raman scattering investigations, it is established that the microheterogeneous environment around the Au nanorods plays a crucial role in driving the interaction of analyte molecules.
\end{abstract}

Keywords. Isothermal titration calorimetry; surface-enhanced Raman scattering; gold nanorods; electrostatic interactions.

\section{Introduction}

With the advent of nanotechnology, there is a large interest in the fabrication of hybrid organic-inorganic nanostructured materials for application in material science and biomedical field. ${ }^{1-3}$ Gold nanoparticles (AuNPs) modified with suitable functional molecules, find applications in diverse fields such as sensors, drug delivery, biodiagnostics and in electronics. ${ }^{4,5}$ The remarkable efficacy and adaptability of these hybrid nanosystems are attributed to the nature of the interaction between the metal nanoparticles (NPs) and analyte molecules. Hence, understanding of the differential affinity of various molecules on to the NP surface

\footnotetext{
*For correspondence

$\S$ Dedicated to Professor M V George on the occasion of his $90^{\text {th }}$ Birth Anniversary.
}

is of prime importance in the development of hybrid functional nanomaterials. Previous studies on the NPmolecular interactions were focused on the use of techniques such as Fourier transform infrared (FTIR), nuclear magnetic resonance (NMR) and reflection absorption infrared (RAIR) spectroscopies. ${ }^{6,7}$ Moreover, most of the studies in this direction are limited to spherical metal NPs. With the advancement in the synthesis of metal NPs, anisotropic nanomaterials have outperformed and substituted spherical NPs ${ }^{8-10}$ Considering the fact that there are only very few studies focusing on the interaction between anisotropic metal nanostructures and binding molecules, a systematic investigation in this direction is of extreme importance.

Isothermal titration calorimetry (ITC) is a unique technique that can directly measure the binding energetics of different chemical and biological processes, including metal-ion binding, host-guest interactions, 
protein-ligand binding, DNA-protein binding, protein-lipid binding and antigen-antibody binding. The technique can precisely determine the Gibbs energy, enthalpy, entropy and heat capacity changes associated with the binding. ${ }^{11-13}$ While the technique has been extensively used in recent years to investigate the binding energetics in various chemical and biological processes, it has been seldom used in the investigations of metal NPs. ${ }^{14,15}$ Ganesh, Sastry and coworkers have used ITC for studying the energetics of DNA bases as well as the corresponding PNA base monomers interaction with AuNPs. ${ }^{16}$ Rao and coworkers utilized the technique to study the mechanism of cysteine and 3-mercaptopropionic acid induced linear assembly of Au nanorods (AuNRs). ${ }^{17}$ Herein, we use a combination of ITC ${ }^{18-20}$ and surface-enhanced Raman scattering (SERS) ${ }^{21-23}$ to study the interaction of molecules with the surface of AuNRs. While ITC helps in analyzing the nanorod (NR) molecule interactions, SERS can function as a molecular sensing platform. A thiol-bearing molecule, 4-mercaptopyridine is used to study the covalent type interactions, whereas a positively charged molecule, methyl viologen is used to investigate the electrostatic interactions with AuNR surface.

\section{Experimental}

\subsection{Materials and methods}

Solvents and reagents used are purified and dried by standard methods. Photophysical studies are carried out using spectroscopic grade solvents. Stock solutions of 4-mercaptopyridine and methyl viologen are prepared in acetonitrile and water, respectively. Raman and SERS spectra are recorded using a HR800 LabRAM confocal Raman spectrometer operating at $20 \mathrm{~mW}$ laser power using a peltier cooled CCD detector. Raman spectra are collected in a quartz cuvette using a $\mathrm{He}-\mathrm{Ne}$ laser source having an excitation wavelength of 633 $\mathrm{nm}$ and with an acquisition time of $10 \mathrm{~s}$ using a $5 \mathrm{x}$ objective. The baseline is corrected and the spectra are presented after subtracting the AuNR spectra from the original ones. No noticeable absorption spectral changes are observed for the solution during the measurements, confirming that the AuNRs remain stable in solution even after the exposure to laser beam. ITC is carried out using a Microcal VP-ITC instrument. Doubly distilled water and spectroscopic grade acetonitrile is used for all the ITC studies. All the experiments were carried out at room temperature. In all cases, the heat of dilution can be determined by titrating same concentration of 4-mercaptopyridine/methyl viologen (in water/acetonitrile) to the solvent (water/acetonitrile-water mixture) in the cell in the absence of NRs. Only weak peaks were observed indicating the low heat of dilution due to mixing. In the experiments with NRs, the background signals observed after the saturation of reactant in the cell can be attributed to the heat due to ligand dilution or liquid friction during mixing.

\subsection{Isothermal titration calorimetry}

ITC is based on the principle of dynamic power compensation, wherein the amount of power required for maintaining a constant temperature difference between the sample and the reference cell is measured. ${ }^{18-20} \mathrm{~A}$ small power is continuously applied to the sample cell by the feedback system, to determine the baseline level. Each injection of the syringe solution triggers the binding reaction; depending on the binding affinity and the concentration of reactants in the cell, a certain amount of complex is formed. The molecular interaction is accompanied either by the release of heat (exothermic reaction) or by the absorption of heat (endothermic reaction) which causes a difference in temperature between the sample and the reference cell. Depending on the nature of the reaction, the feedback system either lowers or raises the thermal power applied in order to compensate for the temperature difference. The power compensation after each injection is reflected in the form of a peak in the ITC plot. The amount of heat associated with the injection is obtained by integrating the area under the peak.

\subsection{Synthesis of Au nanorods}

AuNRs were synthesized following the photochemical method. ${ }^{24}$ A growth solution is prepared by dissolving cetyltrimethylammonium bromide $(440 \mathrm{mg})$ and tetraoctylammonium bromide $(4.5 \mathrm{mg})$ in $15 \mathrm{~mL}$ of water in a cylindrical quartz tube (length $15 \mathrm{~cm}$ and diameter $2 \mathrm{~cm}$ ). To this solution, $1.25 \mathrm{~mL}$ of $0.024 \mathrm{M} \mathrm{HAuCl}_{4}$ solution is added along with $325 \mu \mathrm{L}$ of acetone and $225 \mu \mathrm{L}$ of cyclohexane. It is reported that $\mathrm{AgNO}_{3}$ is essential for synthesizing AuNRs and controlling their aspect ratio. To the above solution, varying amounts of $\mathrm{AgNO}_{3}(0.1 \mathrm{M})$ are added for synthesizing AuNRs of different aspect ratios: $2.2(325 \mu \mathrm{L}), 3.0(400 \mu \mathrm{L})$ and $3.7(450 \mu \mathrm{L})$. The quartz tube is closed with a rubber stopper through which a glass rod is inserted $(15 \mathrm{~cm}$ length and $1 \mathrm{~cm}$ diameter). The glass rod helps in reducing the effective thickness of the solution and increases the light absorbance. A photochemical reaction is carried out at $300 \mathrm{~nm}$ irradiation in a Rayonet photochemical reactor for $18 \mathrm{~h}$. AuNRs prepared by a photochemical method are first purified by centrifugation. The residue obtained after 10 min of centrifugation (7000 rpm) is dispersed in $2 \mathrm{~mL}$ of $0.7 \mathrm{M}$ cetyltrimethylammonium bromide (CTAB) solution and kept undisturbed for $12 \mathrm{~h}$. The supernatant solution is carefully decanted and the residue is suspended in water. The solution is kept at $5{ }^{\circ} \mathrm{C}$ for $2 \mathrm{~h}$ to remove excess CTAB. The excess CTAB crystallized out, after cooling, is separated by filtration. The filtrate contains monodisperse AuNRs that are used directly for various studies. 


\section{Results and Discussion}

Various types of interactions that operate between the analyte molecule and the NP substrates include covalent bond formation, hydrogen bonding, electrostatic interactions, van der Waals interaction and $\pi$ stacking. We investigated the strength of various interactions between the analyte molecules and AuNRs by following ITC. In this regard, two types of interactions with AuNRs are probed: (i) covalent binding of a thiol-bearing molecule, namely, 4-mercaptopyridine and (ii) electrostatic interaction of a positively charged molecule, namely, methyl viologen. AuNRs were synthesized by following the photochemical method and excess capping agents were removed (details provided in the experimental section). ITC experiments are carried out in two solvent systems: (i) aqueous solution and (ii) in a mixture of (1:4) of water and acetonitrile at room temperature $\left(25 \pm 1{ }^{\circ} \mathrm{C}\right)$.
An aqueous solution $(40 \mu \mathrm{L})$ of 4 -mercaptopyridine $(10 \mu \mathrm{M})$ is injected in equal steps of $2 \mu \mathrm{L}$ to an aqueous solution of AuNRs $(200 \mu \mathrm{L} ; 0.12 \mathrm{nM}$ particle concentration) taken in an ITC cell. Experiments carried out in aqueous medium show negligible heat change (Figures 1C). In contrast, large heat change is observed when the experiments are carried out in a mixture (1:4) of water and acetonitrile (Figures 1D). The traces presented in Figure 1C, D correspond to raw calorimetric data obtained during titration and the lower plot represents integrated heat response plotted against the concentration of the solution added to the reaction vessel containing the aqueous AuNRs (Figure 1). We observe that the peak intensity decreases with each successive injection due to the binding of molecules to the AuNRs. The number of free sites available on the NR surface decreases with the increase in analyte concentration, thereby reducing the peak intensity.
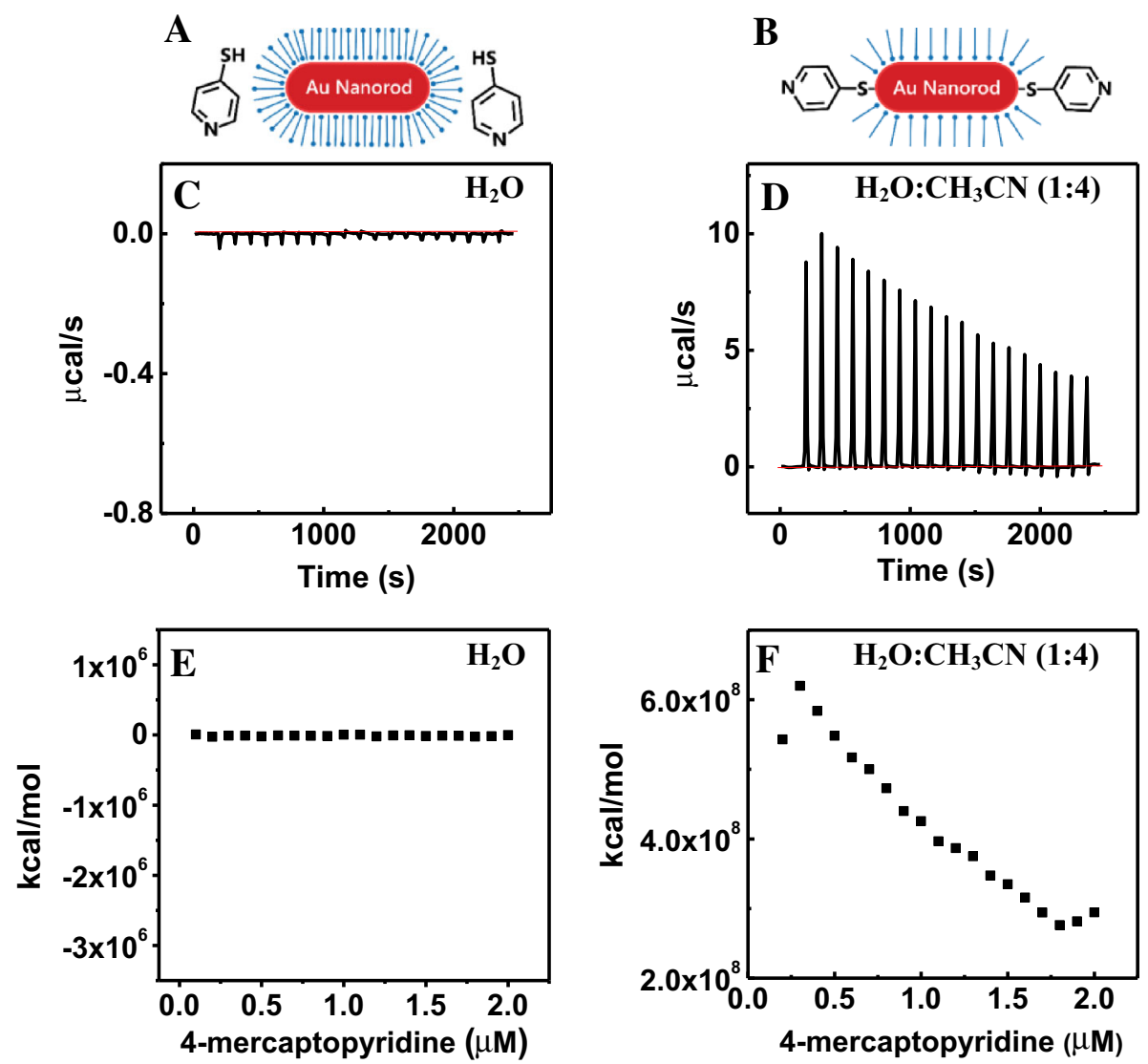

Figure 1. ITC titration illustrating the binding of 4-mercaptopyridine onto AuNRs with an aspect ratio of 2.5. (A, B) Schematic representation of the interaction of 4-mercaptopyridine with the surface of AuNRs in (A) water and (B) mixture (1:4) of water and acetonitrile. (C, D) Raw calorimetric data obtained during injection of 4-mercaptopyridine $(10 \mu \mathrm{M})$ into the calorimetric cell containing AuNRs $(200 \mu \mathrm{L}$; particle concentration of $0.12 \mathrm{nM}$ ) (C) in water and (D) mixture of (1:4) water and $\mathrm{CH}_{3} \mathrm{CN}$. $(\mathrm{E}, \mathrm{F})$ Integrated data of the curves in panels (C) and (D), respectively, are plotted as a function of the concentration of the 4-mercaptopyridine added to the reaction cell. 

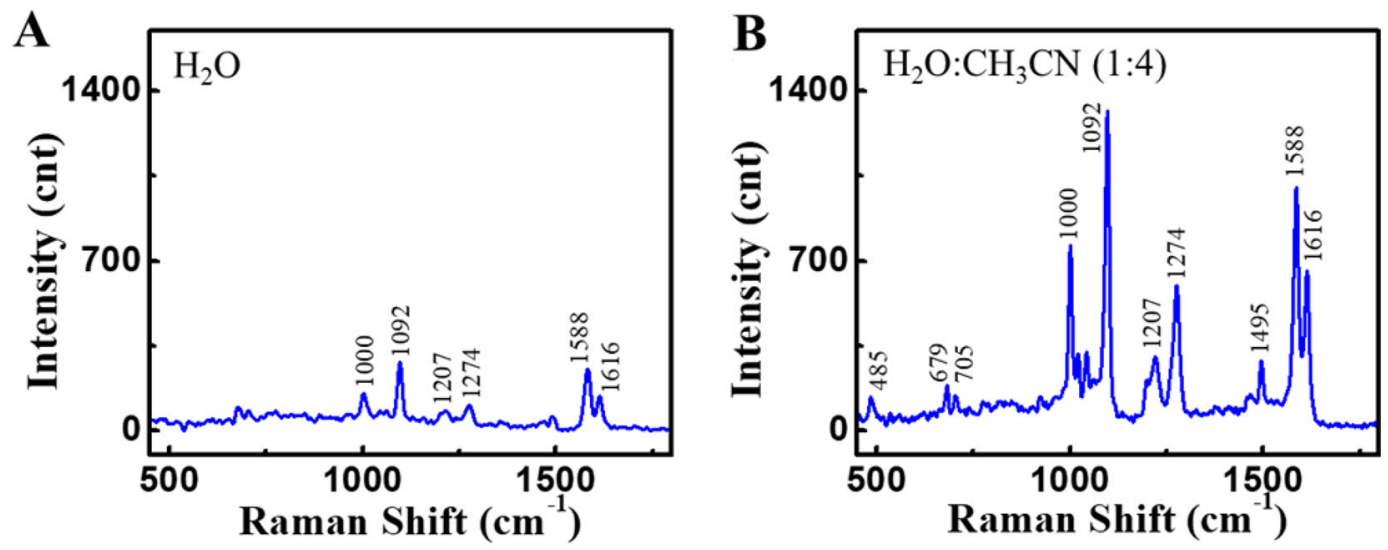

Figure 2. Raman spectra obtained on addition of 4-mercaptopyrine $(0.5 \mu \mathrm{M})$ to AuNRs (particle concentration of $0.12 \mathrm{nM})(\mathrm{A})$ in water and (B) in a mixture (1:4) of water and acetonitrile.

While investigating the interaction of two species in solution, the binding isotherm can be directly plotted against the molar ratio of reactants in solution. However, in the present case, the lack of information on the exact surface area of the NPs (i.e., number of moles of surface $\mathrm{Au}$ atoms) restricts us from plotting the binding isotherms against molar ratio involving the analyte and AuNR. In the present case, the binding isotherms are plotted against the total concentration of the analyte molecule added to the reaction cell (Figures 1E, F). This calorimetric response is merely used to identify the trends in the binding behavior and is not used to derive any thermodynamic parameters to avoid any potential errors that may arise due to the above-cited reasons. ${ }^{25,26}$ The plot of binding isotherm against the concentration of analyte indicates stronger molecular interaction with the surface of NRs in an acetonitrilerich solvent. In contrast, under similar conditions, the analyte-substrate interactions are found to be weak and the heat changes are negligible in an aqueous medium. These results confirm the effective binding of analyte molecules onto the surface of NRs in acetonitrile-rich solvents. In host-guest complexation reactions, favorable enthalpy changes are always balanced by entropic penalties and vice versa, and this is termed enthalpyentropy compensation. ${ }^{14}$ The ITC plot in Figure 1D shows that the calorimetric response during the titration of 4-mercaptopyridine against AuNRs is endothermic. The fact that the interaction is endothermic (which tends to oppose the reaction) suggests that the reaction is entropically driven.

The interactions between the analyte and AuNRs are further probed by following SERS studies. Raman spectra are recorded using a confocal Raman spectrometer by exciting at $633 \mathrm{~nm}$ (He-Ne laser). The
AuNR solutions are kept for 10 min after the addition of 4-mercaptopyridine, and the Raman and extinction spectra are recorded. It is reported that the thiol derivatives preferentially bind onto the $\{111\}$ planes of the AuNRs resulting in the localization of molecules at the edges. CTAB-capped AuNRs suspended in both water and a mixture (1:4) of water and acetonitrile exhibit Raman signals corresponding to the solvent molecules; however, no spectroscopic signals corresponding to the CTAB molecules are observed. AuNRs are found to be stable in both the solvent media even after the addition of higher concentrations of the analyte molecules. It may be noted that Raman signals of 4-mercaptopyridine are not observed in the absence of AuNRs, even at a concentration of $5 \mathrm{mM}$ solution. The Raman spectra obtained on addition of 4-mercaptopyridine $(0.5 \mu \mathrm{M})$ to an aqueous solution of AuNRs (particle concentration $0.12 \mathrm{nM}$ ) showed peaks corresponding to the various molecular vibrational modes of 4-mercaptopyridine (Figure 2A); however, the peaks showed lower intensity. Under similar conditions, intense Raman signals corresponding to the molecular vibrations of 4-mercaptopyridine is observed in a mixture (1:4) containing of water and acetonitrile (Figure 2B). The difference in the peak intensities on changing the solvent composition is a clear indication of the strong interaction in water-acetonitrile mixture compared to that in an aqueous medium. The spectral data and the assignment of peaks corresponding to various vibrational modes of 4-mercaptopyridine are listed in Table $1 .{ }^{27}$ The information on the interaction of analytes on the surface of AuNRs in different solvent compositions obtained from both ITC measurements and Raman spectral studies are in good agreement.

The differential interaction of analyte molecules with AuNR surface in two different solvent compositions 
Table 1. Spectral data and vibrational assignment of 4mercaptopyridine

\begin{tabular}{lc}
\hline SERS peaks $\left(\mathrm{cm}^{-1}\right)$ & Assignment \\
\hline 485 & $\delta(\mathrm{C}-\mathrm{S}) / \gamma(\mathrm{CCC})$ Ring breathing \\
679,705 & $\delta(\mathrm{C}-\mathrm{C}) / \nu(\mathrm{C}-\mathrm{S})$ \\
1000 & $\nu($ ring breathing $)$ \\
$1092,1207,1274$ & $\beta(\mathrm{C}-\mathrm{H})$ \\
1495 & $\nu(\mathrm{C}=\mathrm{C} / \mathrm{C}=\mathrm{N})$ \\
1588 & $\nu(\mathrm{C}-\mathrm{C})$ \\
1616 & $\nu(\mathrm{C}-\mathrm{C})$ Ring stretching \\
\hline
\end{tabular}

can be explained on the basis of varying surface coverage of AuNRs by the capping ligands. Earlier studies from our group have shown that the surface charge on AuNRs can be tuned by varying the solvent composition. ${ }^{28}$ AuNRs are thickly packed by a bilayer of $\mathrm{CTAB}$ in an aqueous medium, and this breaks down into a monolayer with increasing content of organic solvent. ${ }^{28}$ The thick bilayer of CTAB prevents the effective interaction of analyte molecules with the surface of the NRs. This results in a weak binding of analyte molecules with AuNR surface as evident from ITC. On increasing the composition of acetonitrile, the CTAB bilayer breaks into the monolayer, exposing the surface of AuNRs to the solvent environment. In acetonitrile-rich solvents, analyte molecules can easily penetrate through the monolayer of CTAB and interact with the NR edges giving rise to intense Raman signals. A plot of variation in the intensity of the ring breathing mode of 4-mercaptopyrine against varying concentration of the analyte molecule is presented in Figure 3A. A large increase in Raman signal is observed in acetonitrile-rich solvent compared to that in an aqueous medium. TEM images of AuNRs monitored in water and a mixture (1:4) of water and acetonitrile after the addition of 4-mercaptopyridine showed isolated AuNRs dispersed on the surface of the grid (Figures 3B, C). These results indicate that AuNRs remain stable and do not undergo any aggregation under these experimental conditions.

Further, the electrostatic interaction between the positively charged molecule, namely, methyl viologen and AuNRs is probed by following ITC and SERS. The ITC data obtained on addition of methyl viologen in an aqueous medium and in a mixture (1:4) of water and $\mathrm{CH}_{3} \mathrm{CN}$ are presented in Figure 4. An aqueous solution of methyl viologen $(10 \mu \mathrm{M})$ is added in $2 \mu \mathrm{L}$ quantities (20 additions) to AuNRs (concentration $=0.12 \mathrm{nM}$ ) in water kept in the cell. The heat changes after each addition is monitored. Experiments carried out in aqueous medium showed negligible heat change, whereas, a large heat change is observed on addition of the methyl viologen to a solution of AuNRs in a mixture (1:4) of water and $\mathrm{CH}_{3} \mathrm{CN}$ (Figure 4). The raw calorimetric data obtained during analyte-substrate titration shows a clear difference in the interaction of the molecules by varying solvent composition (Figures 4C, D). As in the earlier case with 4-mercaptopyridine, the lack of information on the exact surface area of the NP restricts us from plotting the binding isotherms against molar ratio involving the analyte and AuNRs. ${ }^{25,26}$ Integrated heat responses obtained from the raw data are plotted against the concentration of methyl viologen added to the reaction vessel containing AuNRs (Figures 4E, F). The positive peaks represent heat absorption after each injection of the methyl viologen into the AuNR solution as a function

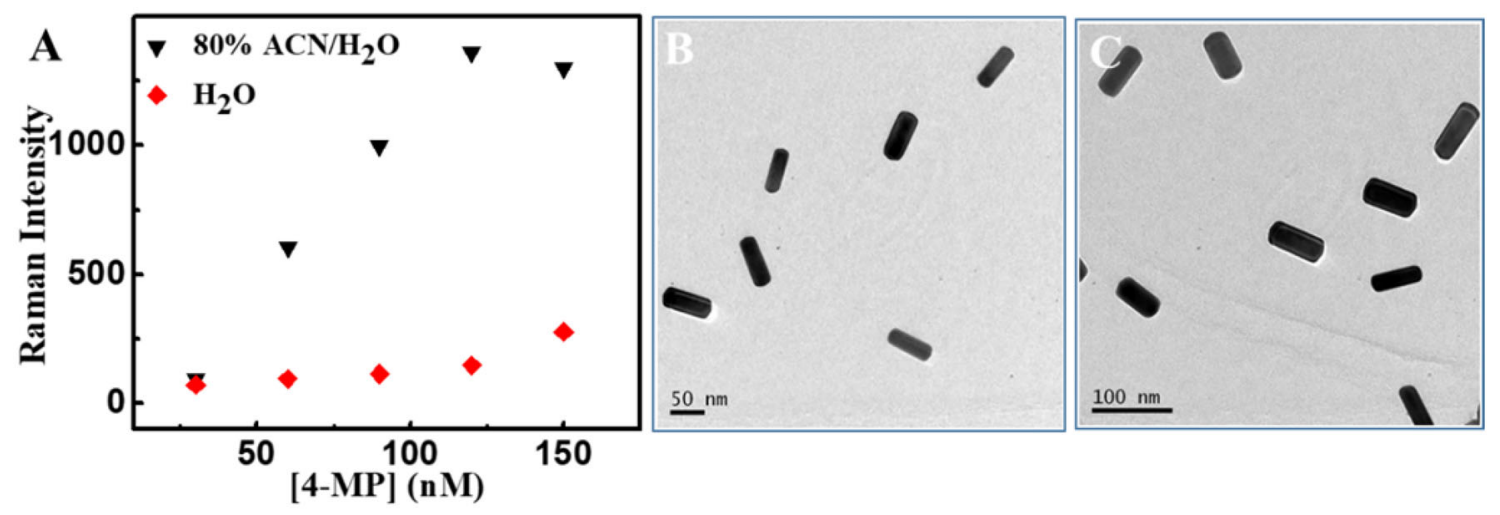

Figure 3. (A) Variation in Raman signal intensity of the ring breathing mode of 4-mercaptopyridine on the addition of varying concentrations of 4-mercaptopyridine to AuNRs (particle concentration of $0.12 \mathrm{nM}$ ) in water as well as in a mixture $(1: 4)$ of water and $\mathrm{CH}_{3} \mathrm{CN}$. (B,C) TEM images of AuNRs (B) in water and (C) in a mixture (1:4) of water and $\mathrm{CH}_{3} \mathrm{CN}$ after the addition of 4-mercaptopyridine. 

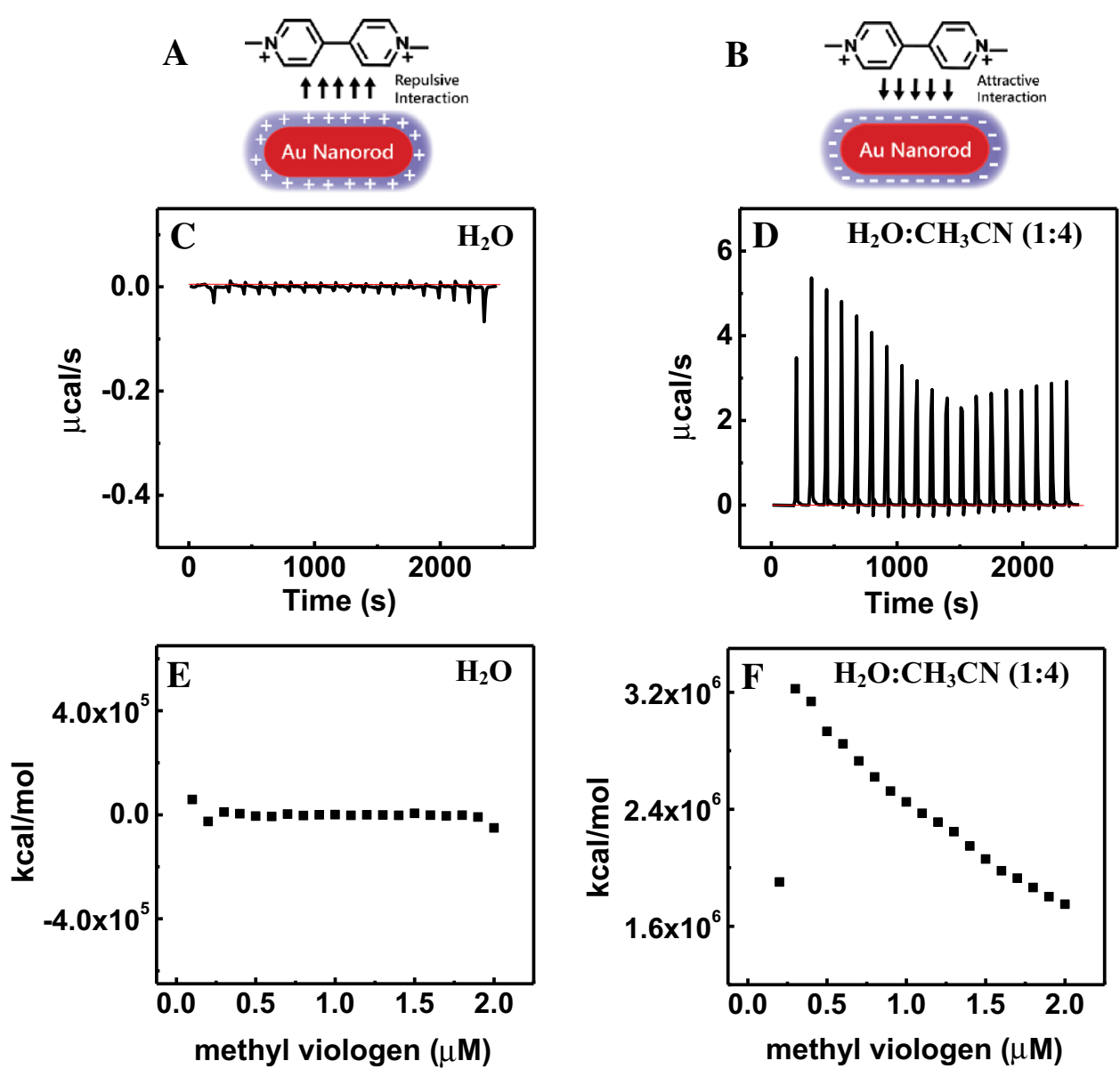

Figure 4. Interaction of methyl viologen with AuNRs followed by ITC. (A, B) Schematic representation of methyl viologen interaction with the surface of AuNRs (A) in water and (B) in a mixture (1:4) of water and $\mathrm{CH}_{3} \mathrm{CN}$. (C, D) Raw calorimetric data obtained during injection of methyl viologen $(10 \mu \mathrm{M})$ into the calorimetric cell containing AuNRs $(200 \mu \mathrm{L}$; particle concentration of $0.12 \mathrm{nM}$ ) (C) in water and (D) a mixture (1:4) of water and $\mathrm{CH}_{3} \mathrm{CN}$. (E, F) Integrated data of the curves in panels (C) and (D), respectively, are plotted as a function of the concentration of methyl viologen solution added to the reaction cell.

of time. The heat changes from the ITC clearly indicate that, in acetonitrile-rich solvents, NRs possess a negative surface charge. The positively charged analyte molecule strongly interacts with the surface of rods. On the other hand, under similar conditions, in an aqueous medium where the NRs possess a positive zeta potential, the analyte-substrate interactions are found to be negligible.

The electrostatic interactions of analyte molecules with AuNRs in water and a mixture (1:4) of water and $\mathrm{CH}_{3} \mathrm{CN}$ are probed by following SERS spectra. Addition of methyl viologen $(1 \mu \mathrm{M})$ to AuNRs (particle concentration of $0.12 \mathrm{nM}$ ) in water shows Raman peaks of low-intensity corresponding to the molecular vibrations of methyl viologen (Figure 5A). Under similar conditions, intense peaks are observed when the solvent system is varied to a mixture (1:4) of water and $\mathrm{CH}_{3} \mathrm{CN}$ (Figure 5B). Apart from the changes in intensity, additional peaks are observed in the SERS spectra of methyl viologen in an acetonitrile-rich solvent (Figures 5A, B). Two plausible reasons can be attributed to this; (i) the SERS effect is very weak in water as the molecule cannot approach the surface of nanorods, and therefore most vibrational signatures are not observed, and/or (ii) there are certain molecular vibrations that are forbidden in normal Raman spectrum, become allowed when the molecule interacts with the NR surface. The differences in SERS spectra clearly indicate that the positively charged analyte molecules interact more effectively with the surface of NRs in a mixture (1:4) of water and $\mathrm{CH}_{3} \mathrm{CN}$ compared to that of the aqueous medium. The 

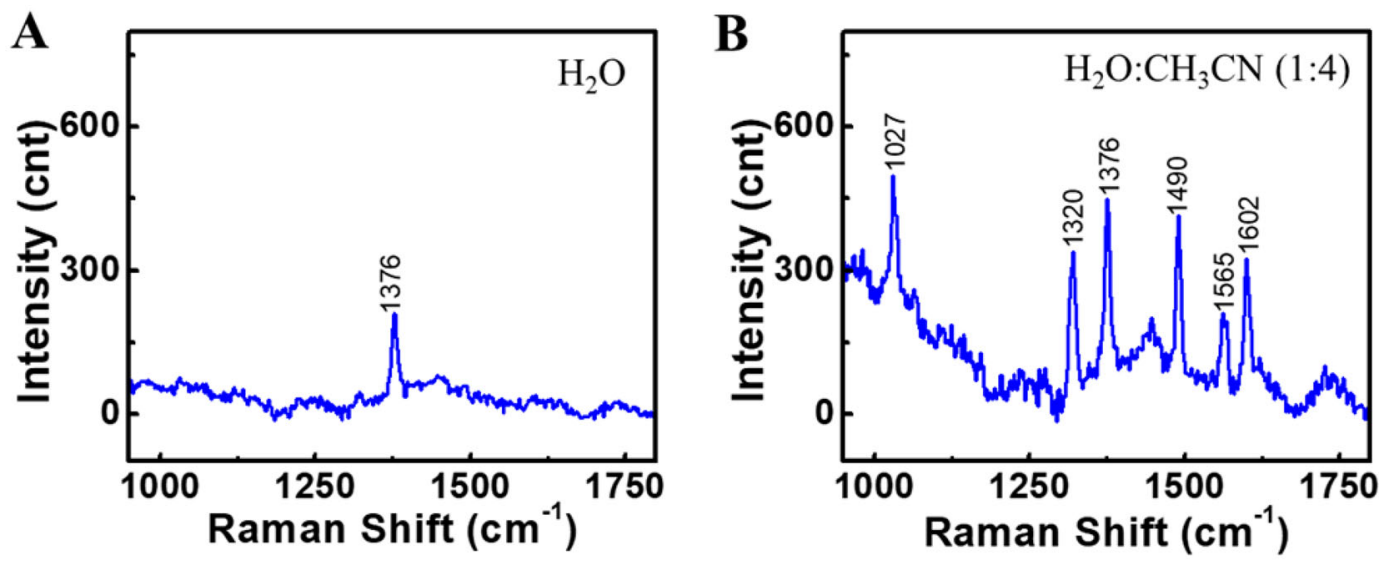

Figure 5. SERS spectra obtained on addition of methyl viologen $(1 \mu \mathrm{M})$ to AuNRs (particle concentration of $0.12 \mathrm{nM})(\mathrm{A})$ in water and (B) in a mixture (1:4) of water and $\mathrm{CH}_{3} \mathrm{CN}$.

Table 2. Raman spectral data and vibrational assignment of methyl viologen.

\begin{tabular}{lc}
\hline SERS peaks $\left(\mathrm{cm}^{-1}\right)$ & Assignment \\
\hline 1027 & $\nu$ (ring breathing $)$ \\
1320 & interring $(\mathrm{C}-\mathrm{C}), \nu(\mathrm{C}-\mathrm{C})$ \\
1376 & $\nu(\mathrm{N}-\mathrm{CH})$ \\
1490 & $\nu(\mathrm{C}=\mathrm{C})$ \\
1565 & $\nu(\mathrm{C} \equiv \mathrm{N})$ \\
1602 & $\nu(\mathrm{C}-\mathrm{C})$, Ring stretching \\
\hline
\end{tabular}

vibrational modes corresponding to different peaks observed in the SERS spectrum of methyl viologen is presented in Table 2. ${ }^{30}$

Further, the interaction of a negatively charged molecule, namely bovine serum albumin (BSA), with AuNRs is studied in water and acetonitrile-rich solvent with the help of ITC. The raw calorimetric data obtained during BSA-AuNR titration exhibit trends opposite to that observed for methyl viologen as the analyte molecule (Figures 6A, B). ITC curves show substantial heat exchange on the addition of BSA to AuNRs in water (Figure 6) whereas weak peaks are observed in an acetonitrile-rich solvent (Figure 6B). These results indicate strong binding of BSA to AuNRs in water and weak interaction with the NR surface in a mixture of acetonitrile and water. The negative peaks in water represent exothermic reaction and the extent of exothermicity decreases after each addition of the analyte indicating saturation of the available site for binding. The heat changes from the ITC indicate that in an aqueous medium the NRs possess a positive charge which enables effective interaction with the negatively charged analyte molecule.
It is reported that AuNRs suspended in water are covered by a bilayer of CTAB molecules. ${ }^{29}$ The excess positive charge originates from the polar head groups of CTAB molecules and hence resulting in a positive zeta potential $(\zeta)$ in water. In contrast, with an increase in the composition of organic solvent, the bilayer structure collapses to a monolayer, resulting in the loss of CTAB from the surface of the AuNRs and leads to a decrease in $\zeta$ value. ${ }^{28} \mathrm{~A}$ charge reversal from positive to negative $\zeta$ was observed with further increase in $\mathrm{CH}_{3} \mathrm{CN}$ content. The NRs exhibited a positive $\zeta$ of $+29.8 \mathrm{mV}$ in water and negative $\zeta$ of $-27.4 \mathrm{mV}$ in a mixture (1:4) of acetonitrile and water. In acetonitrilerich solvents, the number of CTAB molecules protecting the AuNRs may be much lower and the inherent negative surface on AuNRs contribute to high negative $\zeta$ value. The stability of AuNRs in $\mathrm{H}_{2} \mathrm{O}$ and acetonitrilerich solvents may be attributed to the large positive and negative $\zeta$ values in the respective solvent environments. AuNRs that possess a positive $\zeta$ in water experience a repulsive interaction with positively charged methyl viologen molecules. As a result, the heat change observed is negligible ruling out the possibility of any interaction and the weak Raman signals further confirm this. Under similar conditions, the addition of the methyl viologen to AuNRs in a mixture (1:4) of water and acetonitrile leads to the strong electrostatic attraction between the positively charged analyte and the negatively charged SERS substrate. These strong interactions give rise to intense signals in the SERS spectrum. Opposite trends are observed on using a negatively charged analyte molecule such as BSA; the molecule effectively interacts with the NR surface in water whereas interactions are found to be weak in an acetonitrile-rich solvent. 

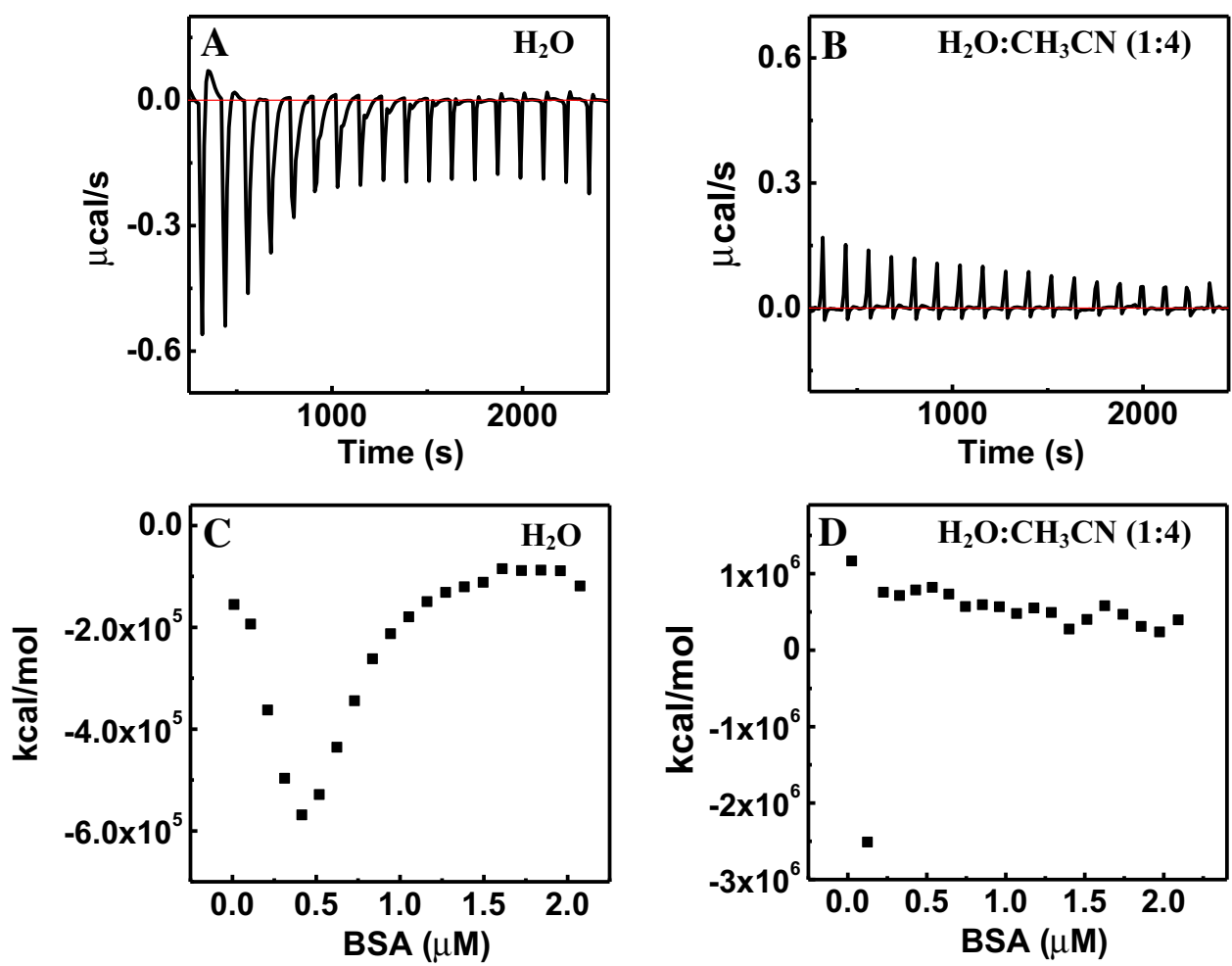

Figure 6. Interaction of BSA with AuNRs followed by ITC. (A, B) Raw calorimetric data obtained during injection of BSA into the calorimetric cell containing AuNRs $(200 \mu \mathrm{L}$; particle concentration of $0.12 \mathrm{nM})(\mathrm{A})$ in water and $(\mathrm{B})$ in a mixture (1:4) of water and $\mathrm{CH}_{3} \mathrm{CN}$. (C, D) Integrated data of the curves in panels (A) and (B), respectively, are plotted as a function of the concentration of BSA solution added to the reaction cell.

\section{Conclusions}

In summary, interactions of analyte molecules on the surface of AuNRs are probed using ITC and SERS. The binding of thiol-bearing analyte molecules on nanorods is facilitated by the breakdown of CTAB bilayer to a monolayer in organic-rich solvent systems. Detailed investigations on the surface charge of NRs revealed that AuNRs have high positive and negative zeta potentials in water and acetonitrile-rich solvents, respectively. NRs possess a negative surface charge in acetonitrilerich solvents and binds electrostatically with positively charged analyte molecules such as methyl viologen. In contrast, under similar conditions, in an aqueous medium where the NRs possess a positive zeta potential and binds with negatively charged analyte molecules such as BSA. Thus, based on ITC and SERS investigations, it is concluded that the microheterogeneous environment surrounding the NRs plays a crucial role in driving the interaction with analyte molecules. These findings are important in future applications of AuNRs as an efficient sensing platform for the detection of molecules of importance in health, environment, and safety.

\section{Acknowledgements}

JK acknowledges the Council of Scientific \& Industrial Research (CSIR), India, for the fellowship. KGT acknowledges the Department of Science and Technology (DST Nanomission Project; SR/NM/NS-23/2016), Government of India for financial support and the J. C. Bose National Fellowship of DST.

\section{References}

1. Li X, Zhu J and Wei B 2016 Hybrid Nanostructures of Metal/Two-Dimensional Nanomaterials for PlasmonEnhanced Applications Chem. Soc. Rev. 453145

2. Thomas R, Kumar J, George J, Shanthil M, Naidu G N, Swathi R S and Thomas K G 2018 Coupling of Elementary Electronic Excitations: Drawing Parallels Between Excitons and Plasmons J. Phys. Chem. Lett. 9919

3. Motl N E, Smith A F, DeSantisa C J and Skrabalak S E 2014 Engineering plasmonic metal colloids through composition and structural design Chem. Soc. Rev. $\mathbf{4 3}$ 3823

4. Wu X, Xu L, Liu L, Ma W, Yin H, Kuang H, Wang L, Xu C and Kotov N A 2013 Unexpected Chirality of Nanoparticle Dimers and Ultrasensitive Chiroplasmonic Bioanalysis J. Am. Chem. Soc. 13518629 
5. Wu X, Hao C, Kumar J, Kuang H, Kotov N A, LizMarzán L M and Xu C 2018 Environmentally responsive plasmonic nanoassemblies for biosensing Chem. Soc. Rev. 474677

6. Hiromi K, Petrovykh D Y, Tarlov M J and Whitman L J 2003 Base-Dependent Competitive Adsorption of Single-Stranded DNA on Gold J. Am. Chem. Soc. 125 9014

7. Demers L M, Ostblom M, Zhang H, Jang N H, Liedberg B and Mirkin C A 2002 Thermal Desorption Behavior and Binding Properties of DNA Bases and Nucleosides on Gold J. Am. Chem. Soc. 12411248

8. Liz-Marzán L M and Grzelczak M 2017 Growing Anisotropic Crystals at the Nanoscale Science 3561120

9. Chen H, Shao L, Li Q and Wang J 2013 Gold Nanorods and their Plasmonic Properties Chem. Soc. Rev. 422679

10. Weiner R G, Kunz M R and Skrabalak S E 2015 Seeding a New Kind of Garden: Synthesis of Architecturally Defined Multimetallic Nanostructures by SeedMediated Co-Reduction Acc. Chem. Res. 482688

11. Baier G, Costa C, Zeller A, Baumann D, Sayer C, Araujo P H H, Mailander V, Musyanovych A and Landfester K 2011 BSA Adsorption on Differently Charged Polystyrene Nanoparticles using Isothermal Titration Calorimetry and the Influence on Cellular Uptake Macromol. Biosci. 11628

12. Krishnan R and Gopidas K R $2011 \beta$-Cyclodextrin as an End-to-End Connector J. Phys. Chem. Lett. 22094

13. Trani J M D, Moitessier N and Mittermaier A K 2017 Measuring Rapid Time-Scale Reaction Kinetics Using Isothermal Titration Calorimetry Anal. Chem. 897022

14. You C-C, Agasti S S and Rotello V M 2008 Isomeric Control of Protein Recognition with Amino Acid-and Dipeptide-Functionalized Gold Nanoparticles Chem. Eur. J. 14143

15. Lang B 2010 Hybridization Thermodynamics of DNA Bound to Gold Nanoparticles J. Chem. Thermodyn. 42 1435

16. Gourishankar A, Shukla S, Ganesh K N and Sastry M 2004 Isothermal Titration Calorimetry Studies on the Binding of DNA Bases and PNA Base Monomers to Gold Nanoparticles J. Am. Chem. Soc. 12613186

17. Varghese N, Vivekchand S R C, Govindaraj A and Rao C N R 2008 A calorimetric investigation of the assembly of gold nanorods to form necklaces Chem. Phys. Lett. 450340
18. Moore D E, Goode D R, Seney C S and Boatwright J M 2016 Isothermal Titration Calorimetry Can Provide Critical Thinking Opportunities J. Chem. Edu. 93304

19. Dam T K and Brewer C F 2002 Thermodynamic Studies of Lectin-Carbohydrate Interactions by Isothermal Titration Calorimetry Chem. Rev. 102387

20. Campoy A V, Ohtaka H, Nezami A, Muzammil S and Freire E 2004 Isothermal Titration Calorimetry Curr. Protoc. Cell Biol. 23 17.8.1

21. Kumar J and Thomas K G 2011 Surface-Enhanced Raman Spectroscopy: Investigations at the Nanorod Edges and Dimer Junctions J. Phys. Chem. Lett. 2610

22. Zrimsek A B, Chiang N, Mattei M, Zaleski S, McAnally M O, Chapman C T, Henry A-I, Schatz G C and Van Duyne R P 2017 Single-Molecule Chemistry with Surface- and Tip-Enhanced Raman Spectroscopy Chem. Rev. 1177583

23. Nie S and Emory S R 1997 Probing Single Molecules and Single Nanoparticles by Surface-Enhanced Raman Scattering Science 2751102

24. Kim F, Song J H and Yang P 2002 Photochemical Synthesis of Gold Nanorods J. Am. Chem. Soc. 124 14316

25. Joshi H, Shirude P S, Bansal V, Ganesh K N and Sastry M 2004 Isothermal Titration Calorimetry Studies on the Binding of Amino Acids to Gold Nanoparticles J. Phys. Chem. B 10811535

26. Rautaray D, Mandal S and Sastry M 2005 Synthesis of Hydroxyapatite Crystals Using Amino AcidCapped Gold Nanoparticles as a Scaffold Langmuir 21 5185

27. Chao Y, Zhou Q, Li Y, Yan Y, Wu Y and Zheng J 2007 Potential Dependent Surface Enhanced Raman Scattering of 4-Mercaptopyridine on Electrochemically Roughened Silver Electrodes J. Phys. Chem. C 111 16990

28. Pramod P and Thomas K G 2008 Plasmon Coupling in Dimers of Au Nanorods Adv. Mater. 204300

29. Gao J, Bender C M and Murphy C J 2003 Dependence of the Gold Nanorod Aspect Ratio on the Nature of the Directing Surfactant in Aqueous Solution Langmuir 19 9065

30. Zheng J, Li X, Gu R and Lu T 2002 Comparison of the Surface Properties of the Assembled Silver Nanoparticle Electrode and Roughened Silver Electrode J. Phys. Chem. B 1061019 